\title{
Supercontinuum Generation and Intermodal Four-Wave Mixing in a Step-Index Few-Mode Fibre
}

\author{
S. Perret ${ }^{(1)}$, G. Fanjoux ${ }^{(1)}$, L. Bigot ${ }^{(2)}$, J. Fatome $^{(3)}$, G. Millot ${ }^{(3)}$, J. M. Dudley (1), and T. Sylvestre ${ }^{(1)}$ \\ (1) Institut FEMTO-ST, Département d'Optique, CNRS, Université Bourgogne Franche-Comté, Besançon, France \\ (2) Institut IRCICA, Laboratoire de Physique des Lasers Atomes et Molécules, CNRS, Université de Lille, France \\ (3) Laboratoire Interdisciplinaire Carnot de Bourgogne, CNRS, Université Bourgogne Franche-Comté, Dijon, France
}

The complex spatiotemporal dynamics of nonlinear light propagation in multimode fibers (MMFs) has recently witnessed a renewed interest because of their experimental realization in emerging key areas of laser physics and fiber optics [1]. Specifically, MMFs have a number of linear and nonlinear optical properties that make them very attractive to investigate new spatiotemporal effects fundamentally different from standard single-mode fibers. These include the observation of multimode solitons [2], intermodal four-wave mixing (FWM) [3], geometric parametric instabilities [4], spatial beam self-cleaning [5], and the generation of supercontinuum (SC) light when pumping in the strong normal dispersion regime $[6,7]$.

However, to date most of these recent works have been performed using graded-index MMFs featuring weak modal dispersion. Here we show that step-index few-mode fibers also allows for the achievement of far-detuned intermodal FWM and supercontinuum generation. We demonstrate in particular broadband SC generation from $560 \mathrm{~nm}$ up to $2350 \mathrm{~nm}$ by coupling a Q-switched picosecond microchip laser at $1064 \mathrm{~nm}$ into a $15 \mu \mathrm{m}$-core stepindex germanium-doped silica fiber, designed to support five spatial modes at $1064 \mathrm{~nm}$. It is further shown that multiple cascaded intermodal FWM and Raman processes take place in the fiber with large frequency detuning up to $150 \mathrm{THz}$. The multimode properties of this fiber yield a number of intermodal nonlinear coupling terms and the parametric sideband wavelengths have been obtained from the phase-matching condition for intermodal FWM. A further investigation with another microchip pump laser at $532 \mathrm{~nm}$ reveals the observation of many parametric sidebands due to intermodal FWM involving more than eight spatial modes. The very good agreement between theory and experiment at $532 \mathrm{~nm}$ confirms our fiber step-index model, which allows us to simulate the modal eigenvalue solutions of Helmholtz equation to get the effective index for all modes at any wavelength as well as their modal dispersion.

[1] L. G. Wright, D. N. Christodoulides, F. W. Wise, Nat. Photon. 9, 306 (2015).

[2] Z. Zhu, L. G. Wright, D. N. Christodoulides, and F. W. Wise, Opt. Lett. 20, 4819 (2016).

[3] R. Dupiol, A. Bendahmane, K. Krupa, A. Tonello, M. Fabert, B. Kibler, T. Sylvestre, A. Barthelemy, V. Couderc, S. Wabnitz, and G. Millot, Opt. Lett. 7, 1293 (2017).

[4] K. Krupa, A. Tonello, A. Barthelemy, V. Couderc, B. M. Shalaby, A. Bendahmane, G. Millot, and S. Wabnitz, Phys. Rev. Lett. 18, 183901 (2016).

[5] K. Krupa, A. Tonello, B. M. Shalaby, M. Fabert, A. Barthelemy, G. Millot, S. Wabnitz, and V. Couderc, Nat. Photon. 4, 237 (2017).

[6] M. A. Eftekhar, L. G. Wright, M. S. Mills, M. Kolesik, R. A. Correa, F. W. Wise, and D. N. Christodoulides, Opt. Exp. 8, 9078 (2017).

[7] K. Krupa, C. Louot, V. Couderc, M. Fabert, R. Guenard, B. M. Shalaby, A. Tonello, D. Pagnoux, P. Leproux, A. Bendahmane, R. Dupiol, G. Millot, and S. Wabnitz, Opt. Lett. 5785 (2016). 